\title{
Maturation-induced down-regulation of MFG-E8 impairs apoptotic cell clearance and enhances endotoxin response
}

\author{
MICHAEL MIKSA, DHRUV AMIN, RONGQIAN WU, ASHA JACOB, MIAN ZHOU, \\ WEIFENG DONG, WENG-LANG YANG, THANJAVUR S. RAVIKUMAR and PING WANG
}

\begin{abstract}
Center for Immunology and Inflammation, The Feinstein Institute for Medical Research and Department of Surgery, North Shore University Hospital and Long Island Jewish Medical Center, Manhasset, NY 11030, USA
\end{abstract}

Received August 22, 2008; Accepted September 26, 2008

DOI: 10.3892/ijmm_00000080

\begin{abstract}
In sepsis, phagocytosis and the killing of bacteria by phagocytes are important. Similarly, the clearance of accumulating apoptotic cells is critical in maintaining normal immunity. Upon maturation, peritoneal macrophages (PM) become a major source of proinflammatory cytokines, while losing their efficacy of phagocytosis. However, the underlying mechanism remains unknown. Here we investigated the differential effects of apoptotic thymocytes (AoTC) on TNF- $\alpha$ release in immature thioglycolate-elicited PM (TGPM) and mature resident PM (RPM) in vitro by culturing them with or without AoTC and/or LPS. MFG-E8 expression was assessed using Western blotting and the ability to engulf AoTC was determined histologically. Cytokine secretion was measured by ELISA. MAP kinase phosphorylation was assessed using Western blotting. Mature RPM express $<50 \%$ of TGPM MFG-E8 levels and have a $30 \%$ lower capacity to clear AoTC. The proinflammatory response (TNF- $\alpha$ release) to LPS is 5 times higher, and the capability to phagocytose is decreased along with further down-regulation of MFG-E8 after LPS-stimulation. RPMs also lack phagocytosis-induced inhibition of TNF- $\alpha$ release after LPS stimulation. LPSinduced phosphorylation of ERK1/2, p38 and JNK is more enhanced in RPM compared to TGPM. MFG-E8-mediated apoptotic cell phagocytosis results in an inhibition of MAPK and NFאB signaling pathways. Differential MAPK activation may play a role in the enhanced LPS responsiveness of RPM and the lack of MFG-E8 impedes post-phagocytic suppression of LPS-response through the inhibition of those signaling pathways. These results provide a potential mechanistic insight into the benefit of promoting apoptotic cell clearance via MFG-E8 under inflammatory conditions.
\end{abstract}

Correspondence to: Dr Ping Wang, The Feinstein Institute for Medical Research, 350 Community Drive, Manhasset, NY 11030, USA

E-mail: pwang@nshs.edu

Key words: phagocytosis, macrophages, TNF- $\alpha$

\section{Introduction}

Under normal conditions, resident peritoneal macrophages (RPMs) inhabiting the peritoneal cavity, are morphologically determined by numerous lamellipodia, subsurface vacuoles and an irregular eccentric nucleus with heterochromatin margination surrounded by a large number of lysosomes (1). Within $24 \mathrm{~h}$ after intraperitoneal injection of thioglycolate, two new subpopulations of macrophages emerge that can be distinguished morphologically as monocyte-like macrophages with a kidney-shaped nucleus and numerous lysosomes and the large irregular 'inflammatory macrophages' with many filopodia and cytoplasmic inclusions $(1,2)$. Cytochemically these so-called exudate macrophages have a peroxidase activity limited to their lysosomes, while RPMs show abundant activity in the nuclear envelope and rough endoplasmic reticulum. Thioglycolate elicited peritoneal macrophages (TGPMs) have a higher antibody-dependent and -independent endocytotic and exocytotic activity but are less efficient than RPMs in antibody dependent cell-mediated cytolysis and resistance to Listeria monocytogenes infection (3-6). In 1994, Blasi et al discovered that cytokine mRNA transcription and activity are lower in TGPMs than those in RPMs after stimulation with either Candida albicans or LPS (7). Hanayama et al found that TGPMs as well as immature dendritic cells and marginal zone macrophages of the spleen contain abundant milk fat globule epidermal growth factor-factor VIII (MFG-E8)(8). MFG-E8 specifically binds to phosphatidylserine and acts as an opsonin for apoptotic cells by bridging phosphatidylserine on apoptotic cells to integrins on phagocytes $(9,10)$. This aids to enhance the clearance of apoptotic cells, ultimately leading to suppression of proinflammatory responses that are crucial in autoimmunity (8) and sepsis (11). As mature RPMs have a lower capacity for phagocytosis than TGPMs, we speculated that this was also true for the engulfment of apoptotic cells. We therefore hypothesized that RPMs contain lower amounts of MFG-E8 and thus are less efficient to clear apoptotic cells. We further hypothesized that this is associated with an increased responsiveness to LPS.

\section{Materials and methods}

Peritoneal macrophage culture. Rat peritoneal macrophages (RPMs) were isolated by peritoneal lavage $72 \mathrm{~h}$ after i.p. 
injection of $3 \%$ thioglycolate (TGPMs, $30 \mathrm{ml} / \mathrm{kg}$ ) or without pretreatment and cultured in DMEM containing 10\% FBS at $37^{\circ} \mathrm{C}$. Cells were then incubated with LPS (E. coli 055:B5) with or without apoptotic thymocytes. All experiments were performed in accordance with the NIH Guidelines for the Use of Experimental Animals and approved by the IACUC of The Feinstein Institute for Medical Research.

MFG-E8 Western blotting. Macrophages were lysed and homogenized in $1 \mathrm{ml}$ of lysis buffer $(10 \mathrm{mM}$ Tris-buffered saline, $1 \mathrm{mM}$ EDTA, $1 \mathrm{mM}$ EGTA, $2 \mathrm{mM}$ sodium orthovanadate, $0.2 \mathrm{mM}$ PMSF, $2 \mu \mathrm{g} / \mathrm{ml}$ leupeptin, $2 \mu \mathrm{g} / \mathrm{ml}$ aprotinin, and $1 \%$ Triton $\mathrm{X}-100$ ) for $30 \mathrm{~min}$ on ice and tissue sample lysate was cleared by centrifugation at $400 \mathrm{x}$ g for $15 \mathrm{~min}$ at $4^{\circ} \mathrm{C}$. Samples were dissolved in $1 \%$ SDS and quantified using the DC Protein Assay (Bio-Rad, Hercules, CA). Plasma samples were ultrafiltered with Centricon 100 (Millpore) and elute was concentrated x30 using Centricon YM30 filters. Ten $\mu \mathrm{g}$ of protein or $5 \mu \mathrm{l}$ of plasma concentrate was fractionated on a $4-12 \%$ Bis-Tris gel and transferred to $0.2-\mu \mathrm{m}$ nitrocellulose membrane. Nitrocellulose blots were blocked by incubation in TBST $(10 \mathrm{mM}$ Tris- $\mathrm{HCl}, \mathrm{pH} 7.5$, $150 \mathrm{mM} \mathrm{NaCl}, 0.1 \%$ Tween-20) containing $10 \%$ bovine serum albumin for $1 \mathrm{~h}$. Blots were incubated with goat antiMFG-E8 IgG clone G-17 (1:100, Santa Cruz Biotechnology, Santa Cruz, CA; an antibody raised against a 17 amino acid peptide sequence shared by human, mouse and rat MFG-E8 in the $\mathrm{C} 1$ domain) overnight at $4^{\circ} \mathrm{C}$ and then washed vigorously 3 times in TBST 10 for min. Blots were incubated with horseradish peroxidase-labeled rabbit anti-goat IgG for $1 \mathrm{~h}$ at room temperature, washed as before, incubated with ECL (Amersham) and exposed on radiograph film.

Phagocytosis assay. Macrophages were cultured at $2.5 \times 10^{4} /$ well in a 16 -well chamber slide (Nalge Nunc, Rochester, NY). Thymocyte apoptosis was induced by $10 \mu \mathrm{M}$ dexamethasone for $24 \mathrm{~h}\left(>99 \%\right.$ Annexin $\left.^{+} \mathrm{CD} 90^{+}\right)$. After washing with PBS, macrophages were incubated with AoTC at a ratio of 4:1 (AoTC/macrophages) for $90 \mathrm{~min}$. Adherent macrophages were washed with PBS, fixed with $4 \%$ paraformaldehyde, stained with TUNEL and analyzed by fluorescent microscopy using a Nikon Eclipse E600 microscope. Results presented as a ratio of apoptotic cells/ macrophages (phagocytosis index).

Cytokine assay. TNF- $\alpha$ release was quantified in supernatants using a commercial ELISA kit (BD Pharmingen).

Phosphoprotein and nuclear NF $\mathrm{NB}$ Western blots. Macrophages were incubated with apoptotic cells for $90 \mathrm{~min}$ followed by washing with PBS and stimulation with LPS for $15 \mathrm{~min}$. Cells were washed, lysed with whole cell lysate buffer (MAPK), or relaxation and nuclear extraction buffer $(\mathrm{NF} \kappa \mathrm{B}$ and $\mathrm{I} \kappa \mathrm{B} \alpha)$. Protein $(20 \mu \mathrm{g})$ was fractionated on a BisTris gel and Western blotting was performd as described above, using specific antibodies against phospho-p38 and total p38, phospho-JNK and total JNK, phospho-ERK1/2 and total ERK1/2 (Cell Signaling, Danvers, MA), as well as anti$\mathrm{NF \kappa B}$ and $\mathrm{I} \kappa \mathrm{B} \alpha$ antibodies (Santa Cruz Biotechnology, Santa Cruz, CA).
Statistical analysis. Data are expressed as mean \pm SEM and compared by Student t-test or ANOVA and StudentNewman-Keuls (SNK) test. Statistical significance was set at $\mathrm{P}<0.05$.

\section{Results}

We isolated resident peritoneal macrophages (RPM) from the peritoneal lavage fluid of normal rats or thioglycolate-induced peritoneal macrophages (TGPM) 3 days after injection of $10 \mathrm{ml}$ $0.3 \%$ thioglycolate into the peritoneum. The initial lavage population was $>90 \%$ RPMs and $\sim 50 \%$ TGPM (the other half being $\sim 50 \%$ neutrophils and sporadic eosinophils and basophils). After enrichment by adherence, both populations were $>95 \%$ pure (Fig. 1A). MFG-E8 expression analysis by Western blot showed that RPMs contained $<50 \%$ of TGPM MFG-E8 levels (Fig. 1B). Similarly, their ability to phagocytose apoptotic cells was reduced by $30 \%$ (Fig. 1C and D).

To investigate the changes of MFG-E8 expression in RPM after endotoxin challenge, we stimulated the cells in vitro with increasing doses of LPS and measured MFG-E8 expression using Western blotting. Ten $\mathrm{ng} / \mathrm{ml}$ LPS was sufficient to significantly reduce MFG-E8 levels by $45 \%$ with no further reduction after stimulation with $100 \mathrm{ng} / \mathrm{ml}$ LPS (Fig. 2A). As expected, the ability to phagocytose apoptotic cells further decreased in RPMs and was consistently lower than that of TGPM under the same conditions (Fig. 2B).

To demonstrate the difference in cytokine response in TGPM and RPM, we measured TNF- $\alpha$ levels released by these cells $4 \mathrm{~h}$ after the stimulation with LPS. While TGPM were responsive to LPS, RPMs showed a 5-fold higher responsiveness to LPS (Fig. 3A). It has been previously demonstrated that apoptotic cell phagocytosis suppresses cytokine responsiveness in phagocytosing macrophages (12). To determine differences in RPM and TGPM-responsiveness after the phagocytosis of apoptotic cells, we measured the respective TNF- $\alpha$ levels. We showed here that the TNF- $\alpha$ release was suppressed in TGPM, here by $40 \%$, and $32 \%$ after the coincubation with apoptotic thymocytes and stimulation with 10 and $100 \mathrm{ng} / \mathrm{ml} \mathrm{LPS}$, respectively (Fig. 3B). In RPM, however, this suppression was not seen (Fig. 3C).

Since this finding was very useful and novel, we sought to determine intracellular pathways involved in this nonresponsiveness to apoptotic-cell mediated TNF- $\alpha$ suppression. We first investigated differences in MAP kinase activation in RPM and TGPM. TGPM showed a robust activation of the three major MAP kinases ERK1/2, p38 and JNK as demonstrated by their phosphorylation (Fig. 4A). By comparison, RPMs showed a stronger increase in ERK1/2, p38 and JNK activation $15 \mathrm{~min}$ after LPS stimulation, supporting the increased TNF- $\alpha$ responsiveness of these cells. We also explored whether MFG-E8 helps to suppress the proinflammatory pathway within RPMs. We hence incubated the macrophages with apoptotic cells, stimulated them with LPS and examined the activation of MAP kinase and NFKB pathways after the exogenous addition of recombinant MFGE8 (rMFG-E8). While apoptotic cells alone had no effect on these pathways, the addition of rMFG-E8 to apoptotic cells prior to phagocytosis and LPS stimulation had a marked suppressive effect on each of the investigated pathways, 

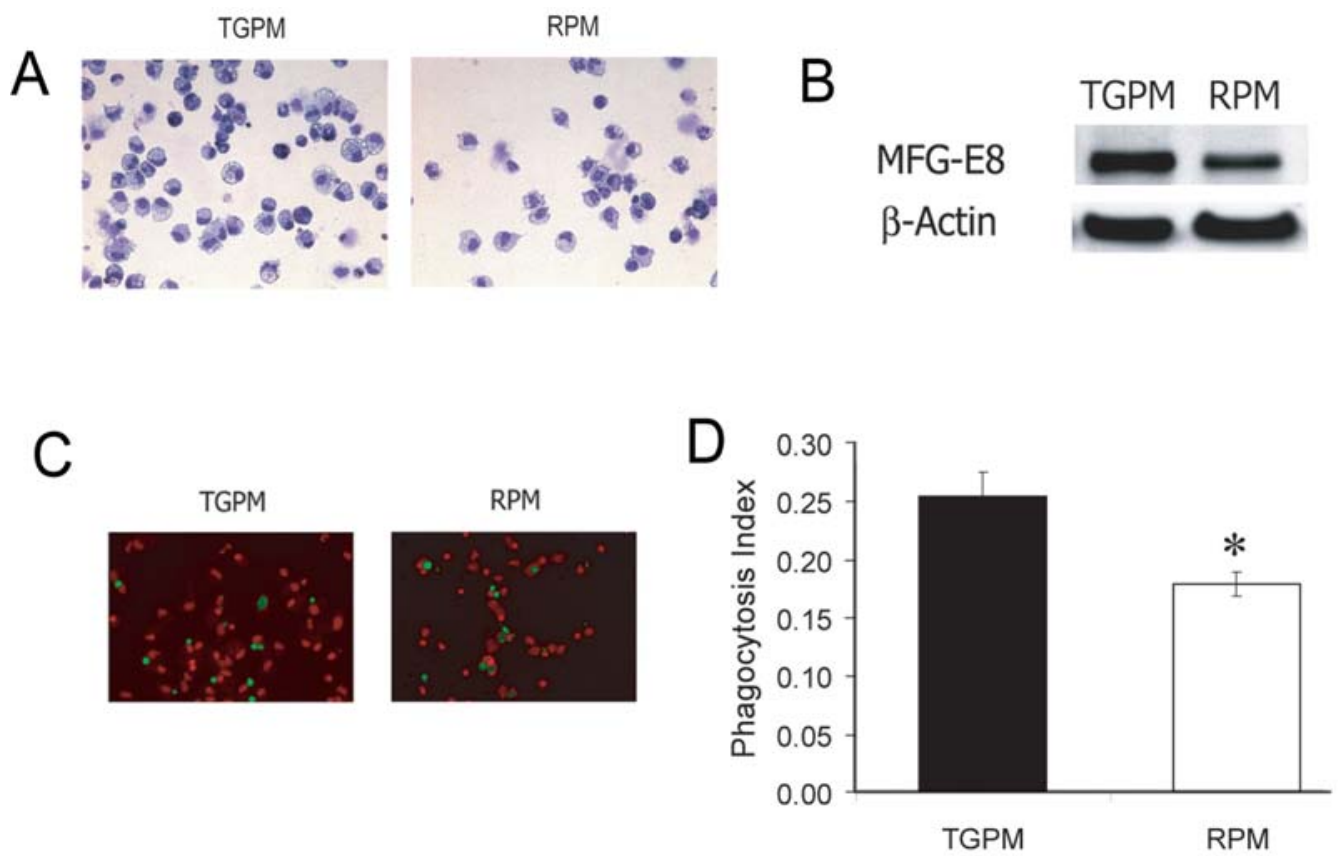

Figure 1. Mature resident peritoneal macrophages (RPM) contain less MFG-E8 than the immature thioglycolate-elicited ( $0.3 \%$ for $72 \mathrm{~h})$ peritoneal (exsudate) macrophages (TGPM) resulting in a reduced efficacy in clearing apoptotic cells. (A) Giemsa-staining of freshly isolated peritoneal macrophages. (B) MFG-E8 Western blot of whole cell lysates. (C) Phagocytosis assay; macrophages were cultured on a chamber slide, fed with 4 apoptotic thymocytes for 90 min, fixed and stained using TUNEL. Red cells depict macrophages and green engulfed apoptotic cells. (D) Quantification of phagocytosis index by calculation engulfed apoptotic cells/macrophages ratio. ${ }^{*} \mathrm{P}<0.05$ vs. TGPM, t-test, $\mathrm{n}=6$.
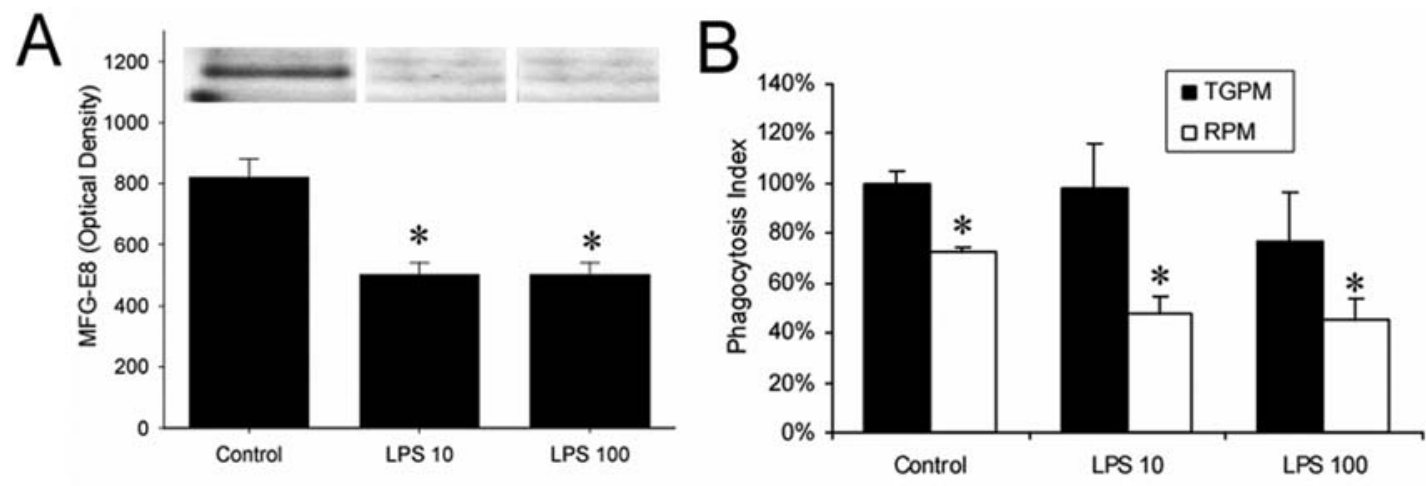

Figure 2. LPS down-regulates MFG-E8 in RPM and further deteriorates apoptotic cell clearance by RPMs compared with TGPMs. (A) RPMs were stimulated with 10 and $100 \mathrm{ng} / \mathrm{ml}$ LPS for $20 \mathrm{~h}$, lysed and protein was used for Western blotting to detect MFG-E8. ${ }^{*} \mathrm{P}<0.05$, ANOVA using SNK test, $\mathrm{n}=3$. (B) TGPMs and RPMs were cultured with or without LPS for $20 \mathrm{~h}$ prior to an efferocytosis assay. Index was normalized to $100 \%$ for non-stimulated TGPM. ${ }^{*} \mathrm{P}<0.05 \mathrm{vs}$. TGPM, ANOVA using SNK test, $\mathrm{n}=6$.

particularly on the p38 and $\mathrm{NF \kappa B}$ pathways that play a key role in the cytokine response of macrophages (Fig. 4B).

\section{Discussion}

In our current study, we demonstrated that TGPMs have higher levels of MFG-E8 and are superior at phagocytosis of apoptotic cells. This leads to an effective suppression of postphagocytic TNF- $\alpha$ release in these macrophages. While RPMs show a stronger activation of proinflammatory intracellular pathways after LPS-stimulation, MFG-E8mediated apoptotic cell phagocytosis suppresses various
ERK1/2, p38, JNK, and NFKB activation, resulting in a lower TNF- $\alpha$ release (Fig. 5).

Macrophages are derived from a myeloid lineage through the differentiation of monoblasts and monocytes that undergo their final differentiation in different tissues, eventually becoming microglia, Kupffer cells, alveolar macrophages or osteoclasts, in the brain, liver, lungs and bone. In these tissues, they assume a central role in the recognition and activation phases of adaptive immune responses. The peritoneal cavity contains a similar cell population of tissuefixed macrophages of which $\sim 10^{7}$ cells can be retrieved by peritoneal lavage in a rat and $\sim 10^{6}$ in a mouse. Over 25 years 

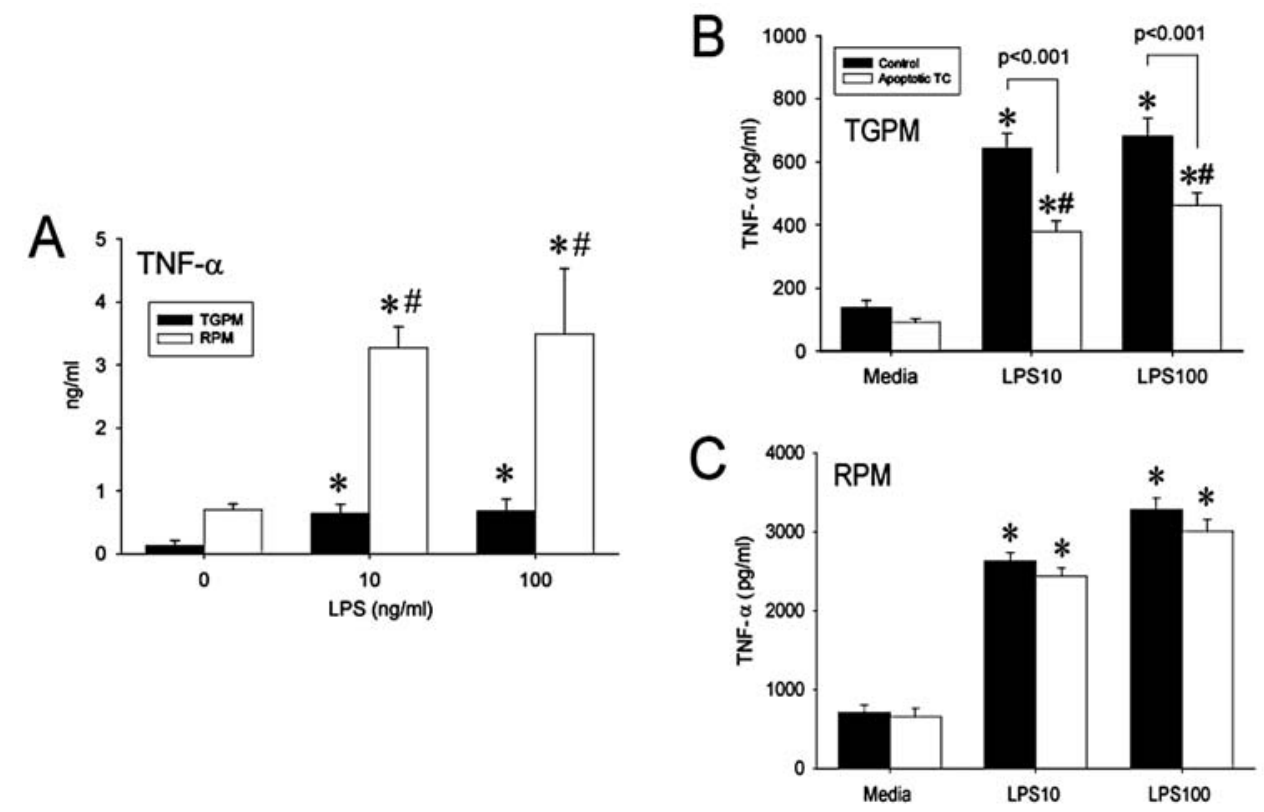

Figure 3. TGPM are less responsive to LPS-stimulated TNF- $\alpha$ release than RPM, which is further suppressed after apoptotic cell phagocytosis. (A) Macrophages were stimulated with LPS for $4 \mathrm{~h}$ and TNF- $\alpha$ release was assessed by measuring concentrations in the supernatant by ELISA. ${ }^{*} \mathrm{P}<0.05 \mathrm{vs} .0 \mathrm{ng} / \mathrm{ml}$ LPS, ${ }^{\#} \mathrm{P}<0.05$ vs. TGPM, two-way ANOVA using SNK test, $\mathrm{n}=10$. (B and C) Macrophages were co-cultured for 90 min with apoptotic thymocytes. Nonphagocytosed apoptotic cells were removed by thorough washing followed by stimulation of the macrophages with LPS for $4 \mathrm{~h}$. TNF- $\alpha$ released from TGPM (B) and RPM (C) was measured by ELISA. ${ }^{*} \mathrm{P}<0.05$ vs. medium, ${ }^{"} \mathrm{P}<0.05$ vs. control, two-way ANOVA using SNK test, $\mathrm{n}=10$.
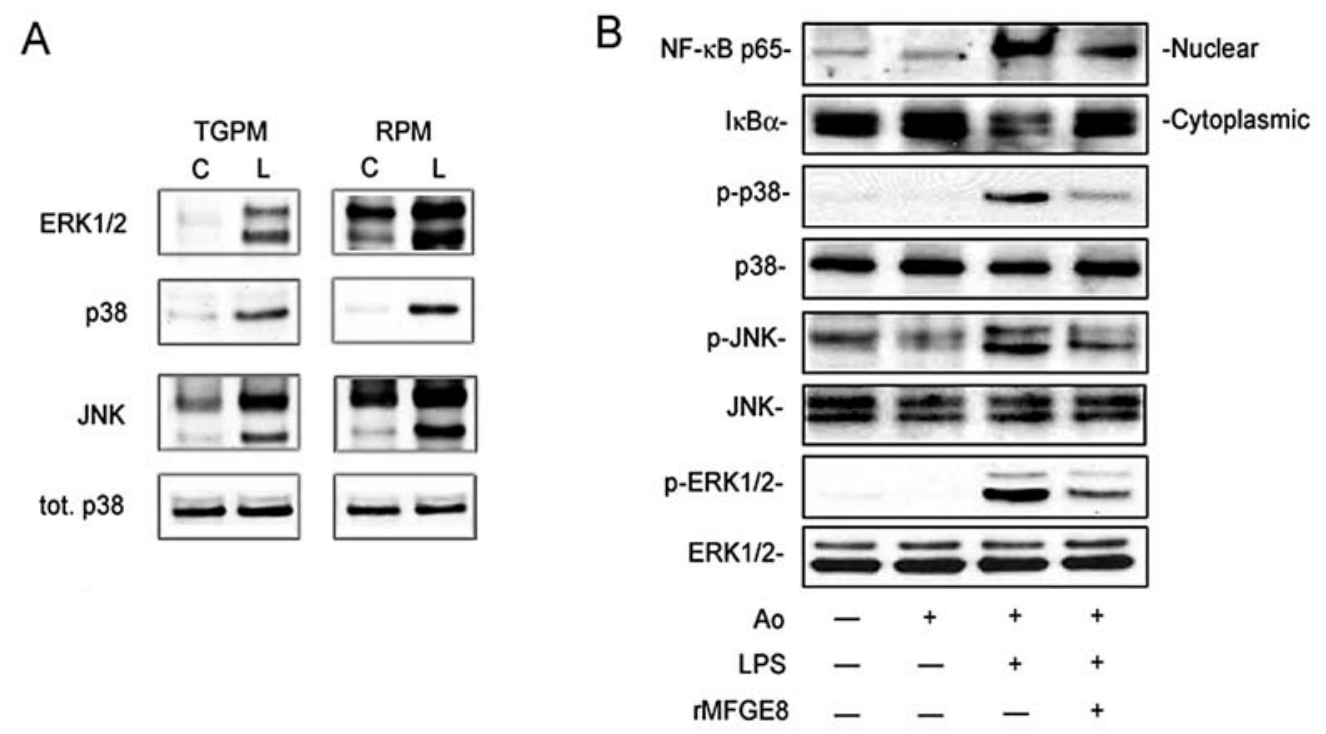

Figure 4. RPMs display a stronger MAPK activation by LPS (A) and apototic cell phagocytosis blocks multiple MAPK and the NFKB pathways in macrophages (B). Macrophages were incubated with apoptotic cells for $90 \mathrm{~min}$ followed by thorough washing and stimulation with $100 \mathrm{ng} / \mathrm{ml} \mathrm{LPS}$ for $15 \mathrm{~min}$. Cells were immediately lysed total or separated into cytoplasmic and nuclear fractions, followed by Western blotting for NFkB (nuclear), IкB $\alpha$ (cytoplasmic), phospho-ERK1/2, phospho-p38 and phospho-JNK. Total MAPK proteins served as internal controls. This figure is representative of three separate experiments with similar results. C, control; L, LPS.

ago researchers were interested in their development and discovered that injection of thioglycolate into the peritoneal cavity leads to an influx of immature monocyte-like (exudate) macrophages that undergo differentiation into mature resident tissue macrophages. This occurs over the course of 3-7 days after thioglycolate stimulation which can be observed through morphological and biochemical changes in these cells (1). The exact mechanism of this influx of cells by an over 70-year old chemical, originally used for depilation and for permanent waves, is still poorly understood, although the involvement of advanced glycation end products (AGEs) are discussed $(13,14)$. AGE has been reported to activate 


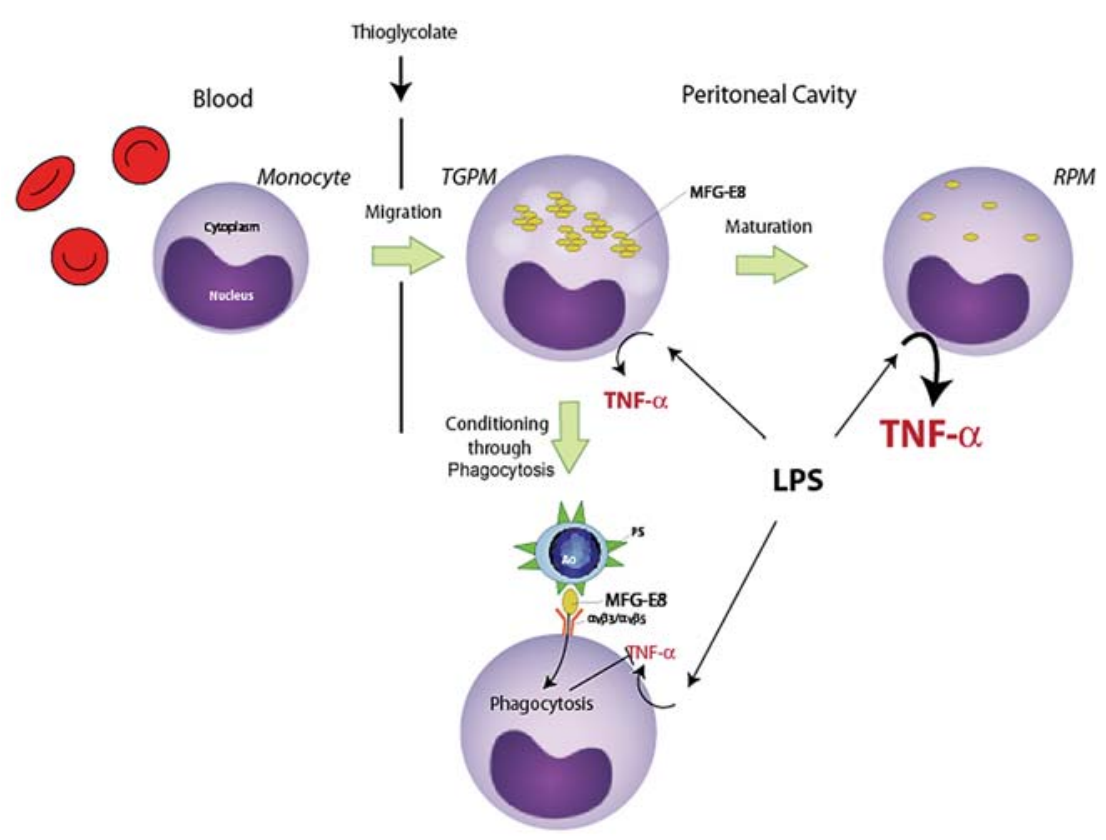

Figure 5. MFG-E8 and apoptotic cell clearance in immature and mature peritoneal macrophages. Thioglycolate recruits immature TGPMs to the peritoneal cavity that contain abundant MFG-E8. RPMs by comparison have less MFG-E8 and are more responsive to LPS stimulation. The apoptotic cell phagocytosis by TGPMs further suppresses LPS-mediated TNF- $\alpha$ release in these cells.

specific receptors, RAGE that induce proinflammatory responses through $\mathrm{NF \kappa B}$ activation (15-17). Thus, AGEs themselves have been shown to elicit opposite effects from those have shown here, especially the increase of TNF- $\alpha$ release and suppression of phagocytic ability $(18,19)$, indicating that AGEs may not be the crucial chemical component in the thioglycolate containing Brewer's medium.

Macrophages play a key role in the innate response and in the orchestration of an adaptive immune response, by releasing proinflammatory mediators that recruit other immune cells to the site of infection and by processing and presenting antigen to lymphocytes eventually mediating a specific host response. It appears, however, that these roles are not performed by a single phenotypic cell population but that these macrophages are, in a sense, specialized to perform certain tasks. For example, it was recognized that TGPMs were less effective in bacterial phagocytosis and killing, while RPMs were very effective to do so $(3,20)$. Effective killing involves the activation of macrophages and bystander immune cells, which is associated with the release of proinflammatory cytokines such as IFN- $\gamma$ and TNF- $\alpha$. We have demonstrated here that RPMs release five times the amount of TNF- $\alpha$ than the newly recruited and presumably more immature TGPMs. Furthermore, we have shown that the ability to clear apoptotic cells also differs between these two cell populations, with the more immature TGPMs being the major players in this respect. This effect is strongly associated with the expression levels of MFG-E8, a factor that may crucially influence the phagocytic ability of macrophages as previous reports have indicated $(8,21)$. Even the exogenous administration of MFG-E8 is able to enhance the phagocytosis of apoptotic cells by RPMs as has been previously shown $(8,21)$. In this respect, the role of MFG-E8 may be two-fold. It increases the clearance of apoptotic cells and suppresses a proinflammatory response at the same time. We have shown that while TGPMs (abundant in MFG-E8) are very effective in this function, RPMs are not, which also reflects in the inability to suppress a proinflammatory response of RPMs after apoptotic cell exposure. MFG-E8enhanced phagocytosis of apoptotic cells attenuates, as we have shown here, crucial intracellular pathways involved in the proinflammatory response of macrophages, such as p38 and NFkB. Immunosuppressive cytokines, such as TGF- 3 and IL-10, released by the (non-phlogistic) phagocytic macrophages may play an additional role in this mechanism $(8,12)$.

The impact of the difference in functionality is evident in acute inflammatory conditions such as sepsis. There is an entire dynamic orchestration of cell responses that regulate the host defense to intruding microbes. Resident macrophages wear off the pathogens by phagocytosing them and mounting an effective immune response, while newly migrating monocytic macrophages appear days later to 'clean up' i.e., remove cell debris and apoptotic cells. In this later phase an inflammatory response is inefficient and probably even detrimental. In sepsis, for example, the initial surge in inflammatory mediators does not subside but continues to stimulate immune responses that lead to self-inflicted damage, which is one of the reasons responsible for organ failure and morbidity in septic animals and possibly patients (22). We have previously shown that MFG-E8 is downregulated in septic rats, which seriously compromised their ability to clear apoptotic cells and resulted in an increased systemic inflammatory response and morbidity (11). This happens usually within $72 \mathrm{~h}$ of septic onset, at the time point when immature monocytic macrophages, rich in MFG-E8, have not yet been recruited to the site of inflammation. Understanding and controlling the kinetics and dynamics of 
this cellular response may be a future goal to attenuate an overzealous inflammatory response found in sepsis and chronic inflammatory diseases such as auto-immunity.

Our results provided new insight into the role of different macrophage subtypes in apoptotic cell clearance and a mechanism responsible for suppressed inflammation. We conclude that TGPMs, appearing to have an immature phenotype, play a major role in clearing apoptotic cells due to higher MFG-E8 levels, while RPM, which are tissue-fixed and mature, are responsible for a strong proinflammatory response.

\section{Acknowledgements}

This study was supported by NIH grants R01 GM057468, R01 GM053008 and R01 AG028352 to Dr P. Wang.

\section{References}

1. Beelen RH and Walker WS: Dynamics of cytochemically distinct subpopulations of macrophages in elicited rat peritoneal exudates. Cell Immunol 82: 246-257, 1983.

2. Kiss AL, Turi A, Muller N, Kantor O and Botos E: Caveolae and caveolin isoforms in rat peritoneal macrophages. Micron 33: 75-93, 2002.

3. Shaw DR and Griffin FM Jr: Thioglycollate-elicited mouse peritoneal macrophages are less efficient than resident macrophages in antibody-dependent cell-mediated cytolysis. J Immunol 128: 433-440, 1982.

4. Baker LA and Campbell PA: Thioglycolate medium decreases resistance to bacterial infection in mice. Infect Immun 27: 455-460, 1980 .

5. Klostergaard J: Role of tumor necrosis factor in monocyte/ macrophage tumor cytotoxicity in vitro. Nat Immun Cell Growth Regul 6: 161-166, 1987.

6. Van Schie RC, Verstraten RG, Van de Winkel JG, Tax WJ and De Mulder PH: Effect of recombinant IFN-gamma (rIFNgamma) on the mechanism of human macrophage IgG FcRImediated cytotoxicity. rIFN-gamma decreases inhibition by cytophilic human IgG and changes the cytolytic mechanism. J Immunol 148: 169-176, 1992.

7. Blasi E, Puliti M, Pitzurra L, Bartoli A and Bistoni F: Heterogeneous secretory response of phagocytes from different anatomical districts to the dimorphic fungus Candida albicans. Cell Immunol 153: 239-247, 1994.

8. Hanayama R, Tanaka M, Miyasaka K, Aozasa K, Koike M, Uchiyama $\mathrm{Y}$ and Nagata $\mathrm{S}$ : Autoimmune disease and impaired uptake of apoptotic cells in MFG-E8-deficient mice. Science 304: 1147-1150, 2004.

9. Asano K, Miwa M, Miwa K, Hanayama R, Nagase H, Nagata S and Tanaka M: Masking of phosphatidylserine inhibits apoptotic cell engulfment and induces autoantibody production in mice. J Exp Med 200: 459-467, 2004.
10. Hanayama R, Tanaka M, Miwa K and Nagata S: Expression of developmental endothelial locus- 1 in a subset of macrophages for engulfment of apoptotic cells. J Immunol 172: 3876-3882, 2004.

11. Miksa M, Wu R, Dong W, Das P, Yang D and Wang P: Dendritic cell-derived exosomes containing milk fat globule epidermal growth factor-factor VIII attenuate proinflammatory responses in sepsis. Shock 25: 586-593, 2006.

12. McDonald PP, Fadok VA, Bratton D and Henson PM: Transcriptional and translational regulation of inflammatory mediator production by endogenous TGF-beta in macrophages that have ingested apoptotic cells. J Immunol 163: 6164-6172, 1999.

13. Li YM, Baviello G, Vlassara H and Mitsuhashi T: Glycation products in aged thioglycollate medium enhance the elicitation of peritoneal macrophages. J Immunol Methods 201: 183-188, 1997.

14. Chavakis T, Bierhaus A, Al-Fakhri N, Schneider D, Witte S, Linn T, Nagashima M, Morser J, Arnold B, Preissner KT and Nawroth PP: The pattern recognition receptor (RAGE) is a counterreceptor for leukocyte integrins: a novel pathway for inflammatory cell recruitment. J Exp Med 198: 1507-1515, 2003.

15. Bierhaus A, Schiekofer S, Schwaninger M, Andrassy M, Humpert PM, Chen J, Hong M, Luther T, Henle T, Kloting I, et al: Diabetes-associated sustained activation of the transcription factor nuclear factor-kappaB. Diabetes 50: 2792-2808, 2001

16. Crabtree M, Pileggi R, Bhattacharyya I, Caudle R, Perez F, Riley J, Vertucci F and Katz J: RAGE mRNA expression and its correlation with nuclear factor kappa beta mRNA expression in inflamed human periradicular tissues. J Endod 34: 689-692, 2008.

17. Dumitriu IE, Baruah P, Valentinis B, Voll RE, Herrmann M, Nawroth PP, Arnold B, Bianchi ME, Manfredi AA and RovereQuerini P: Release of high mobility group box 1 by dendritic cells controls $\mathrm{T}$ cell activation via the receptor for advanced glycation end products. J Immunol 174: 7506-7515, 2005.

18. Liu BF, Miyata S, Kojima H, Uriuhara A, Kusunoki H, Suzuki K and Kasuga M: Low phagocytic activity of resident peritoneal macrophages in diabetic mice: relevance to the formation of advanced glycation end products. Diabetes 48: 2074-2082, 1999.

19. Rashid G, Luzon AA, Korzets Z, Klein O, Zeltzer E and Bernheim J: The effect of advanced glycation end-products and aminoguanidine on TNFalpha production by rat peritoneal macrophages. Perit Dial Int 21: 122-129, 2001.

20. Leijh PC, Van Zwet TL, Ter Kuile MN and Van Furth R: Effect of thioglycolate on phagocytic and microbicidal activities of peritoneal macrophages. Infect Immun 46: 448-452, 1984.

21. Miksa M, Amin D, Wu R, Ravikumar TS and Wang P: Fractalkine-induced MFG-E8 leads to enhanced apoptotic cell clearance by macrophages. Mol Med 13: 553-560, 2007.

22. Riedemann NC, Guo RF and Ward PA: The enigma of sepsis. J Clin Invest 112: 460-467, 2003. 